\title{
An Inverse Prospect Theory Based-Approach for Linear Ordinal Ranking Aggregation with Its Application in Site Selection of Electric Vehicle Charging Station
}

\author{
Nana Liu \\ Business School, \\ Sichuan University, \\ Chengdu, China \\ liunana_lala@qq.com
}

\author{
Zeshui Xu \\ Business School, \\ Sichuan University, \\ Chengdu, China \\ xuzeshui@263.net
}

\author{
Hangyao $\mathrm{Wu}$ \\ Business School, \\ Sichuan University, \\ Chengdu, China \\ wwwhy1012@163.com
}

\author{
Peijia Ren \\ School of Business, \\ Administration \\ South China University \\ of Technology, \\ Guangzhou, China \\ renpeijia@outlook.com
}

\author{
Fanlin Meng \\ Department of \\ Mathematical Sciences, \\ University of Essex, \\ Colchester, UK \\ fanlin.meng@essex.ac.uk
}

\begin{abstract}
Considering that it is difficult for experts to provide precise preference values for the site selection of electric vehicle charging station in risky environment, this paper develops an approach for linear ordinal ranking aggregation to validly improve the efficiency and accuracy of electric vehicle charging station site selection. At first, the inverse value function of prospect theory is applied to reduce the impact of risk. Then, through combining with the concept of information energy, the experts' weights can be derived. Besides, a consistency constraint is added to the individual ranking-based alternatives' weights deriving model, which can guarantee the consistency degree at an acceptable level. Additionally, a consensus and standard deviation-based model is established to aggregate the alternatives' weights. Finally, a numerical case about the electric vehicle charging station site selection is presented to show the usage of the approach, meanwhile, comparative analysis and sensitivity analysis are also conducted which show the robustness and practicability of the approach.
\end{abstract}

Keywords-linear ordinal ranking aggregation, prospect theory, site selection of electric vehicle charging station

\section{INTRODUCTION}

With the increasing attention on clean energy and the sustainability of environment development, the number of electric vehicles grows rapidly, meanwhile, the site selection for electric vehicle charging station (EVCS) attracts much attention from researchers [1]. Selecting appropriate sites for EVCS plays a more and more important role in recent years [2], and inappropriate siting of EVCS can result in negative influence on the development of electric vehicles, the layout of the city traffic arrangement, etc. [1]. There are few studies related to EVCS site selection. Specifically, Gonzalez et al. [3] modelled the daily temporal and spatial behavior of electric vehicles, based on which they optimized the location of EVCS. Liu et al. [1] optimized the site of EVCS by a two-step screening method, where they considered the environment factors and EVCS service radius. To minimize the integrated cost of charging stations and consumers, Jia et al. [4] established the optimization model for EVCS site selection. Similarly, Wang et al. [5] proposed a multi-objective EVCS planning model, which not only ensure charging service, but also reduce the power losses. Besides, considering the complexity of the site selection process, Guo and Zhao [2] established the index system of EVCS site selection and used fuzzy TOPSIS method to choose the site from the point of multi-criteria. In the existing studies, seldom of them considered the risk factors, e.g., legal and policy risks, social and cultural risks, etc. [6, 7] in the site selection process. Besides, many existing studies focus on the optimizationbased models where many difficult assumptions are required, and experts' evaluations that provide much helpful experience are ignored. Hence, in such a situation, considering that experts have different knowledge backgrounds, it is helpful to make decisions via integrating group opinions, i.e. group decision making (GDM) [8]. Additionally, due to incomplete access to information, the site selection process becomes more uncertain, which brings great challenge for experts to make a comprehensive decision. Generally, experts maybe affected by their psychological states, i.e., they are more sensitive to gains than losses [9]. In addition, the complex decisionmaking environment makes experts difficult to express their preferences by extremely precise information. Taking account of the experts' expression habits, we can classify the information provided by experts into two categories, i.e., cardinal information and ordinal information [10]. With cardinal information, experts are required to make evaluations by a certain degree, which is difficult because of the lack of standards. However, when using ordinal information, such as rankings, which is more flexible and intuitive and the relative preference order of alternatives is much easier to be given. Hence, we hold the view that ranking is more applicable for experts to express their preferences in the EVCS site selection process [10].

Linear ordinal ranking (LOR), i.e., a ranking that arranges all alternatives from best to worst or inversely, is applied extensively and the aggregation of LOR information is one of the most significant aspects in GDM [11, 12].The studies about LOR aggregation can be classified into two main parts, i.e., based on the relationship and numerical calculation. As for the former, Yager [13] created an algorithm that contains three main steps [14], i.e., preference vectors construction, reading sequence definition and ranking aggregation. Then, many researchers developed the Yager's algorithm from different perspectives. For example, Franceschini et. al. [15] perfected the algorithm by considering the experts' weights and the incomparable and missing alternatives in the decisionmaking process. Combining the Yager's algorithm with prospect theory, Liang et. al. [14] introduced a novel method in the risky environment, in which the value function was used to calculated the relative position indicator and the weighting function was used to determine the DMs' weights. Later on, $\mathrm{Xu}$ et. al. [16] proposed the dominating index and the 
dominated index according to the relationships among alternatives, and established two corresponding aggregation algorithms respectively. The final ranking in their work was determined by the interaction of the results from the two algorithms. For the methods based on the numerical calculation, since some of the researchers transformed the rankings into numerical forms, i.e., position values [17], fuzzy preference relations [18], interval utility value [19], et al., some basic operators, such as, calculus summatorius [17], weighted average operator [19], et al., have been used to fuse the rankings. For example, Borda [17] firstly used the alternatives' positions in the ranking to represent the LORs and aggregated rankings through summing up the alternatives' positions. Wang et al. [19] took LORs as one of the constraints in a programming model and estimated the corresponding interval utilities by solving the model. After transforming rankings into interval values, the aggregated utility interval values were derived through the interval weighted average rules. Because transforming rankings into numerical values extends the computability of the rankings, some studies derived the aggregated values by establishing programming models. For instance, Hou et al. [20] established an interactive consensus model to derive an aggregated preference map, and illustrated how to measure disagreement degree and how to adjust the preference map to achieve consensus. Beg and Ahmad [21] took the minimum distance depicted by spearman footrule [22] as the objective function, and then applied the gene algorithm to solve the model. Furthermore, based on the Borda-Kendall distance [13] and the Hausdorff Kendall distance [14], two models with the objectives of minimizing the distance between the aggregated ranking and the individual rankings are constructed respectively.

From the existing studies, we find some points that can be improved: (1) there exist a lot of risk factors e.g., legal and policy risks, social and cultural risks, etc. $[6,7]$ for experts in the site selection for EVCS, but most studies just considered these factors as the normal attributes in multi-attribute decision-making, and ignore their influence on the experts' psychology. Hence, we attempt to use the prospect theory inversely in this paper to reduce the influence; (2) experts' weights play an important role in the aggregation process, but in most of the existing studies, the determination of experts' weights is ignored. Hence, in this paper, we propose a method to determine the experts' weights from the perspective of information energy; (3) most of the existing aggregation models just considered the standard deviation of the individual information rather than the efficiency of the aggregation results. Therefore, in this paper, we attempt to establish a model that put these two objectives together.

In order to solve the problems mentioned above, the following work has been conducted. To reduce the influence of psychological bias for EVCS selection in the risk environment and find the "true value" of the subjective information, we apply the inverse value function in prospect theory to depict the experts' psychological characteristic under risk at first in the paper. Then, inspired by the concept of information energy [23] which can be used to measure the un-fuzziness degree of the evaluation information, we derive the experts' weights from the perspective of the information energy in this paper. The greater the information energy, the more efficient the evaluations. Then, we also extend the model proposed by $\mathrm{Xu}$ [24] by adding a consistency constraint to derive the alternatives' weights, which can guarantee the consistency of information. Further, to aggregate all the alternatives' weights, we establish a consensus and standard deviation-based model that considers the consensus degree and the information energy at the same time.

The rest of the paper is organized as follows: in Section II, we present the basic knowledge. In Section III, the details of the approach are proposed. Then, we set a numerical case about the site selection of EVCS to illustrate the usage of the approach and comparative analysis and sensitivity analysis are shown in Section IV. Finally, we draw a conclusion in Section $\mathrm{V}$.

\section{PRELIMINARIES}

Referring to the definitions in [25, 26], Liu et al. gave the definition of the LOR in their technical report.

Definition 1. Given a finite alternative set denoted by $\left\{A_{1}, A_{2}, \cdots, A_{i}, \cdots A_{n}\right\}$, if the relationship between each pair of alternatives belongs to the set $\{\succ, \sim, \prec\}$, and all the alternatives are ranked from the best to the worst or inversely, then we call the ranking $O$ a linear ordinal ranking. There into, " $A_{i} \succ A_{k}$ " means that $A_{i}$ is better than $A_{k}$, " $A_{i} \prec A_{k}$ " means that $A_{i}$ is worse than $A_{k}$, and " $A_{i} \sim A_{k}$ " means that alternative $A_{i}$ is the same to $A_{k}$.

To represent LORs better, referring to the idea of accounting for the number of alternatives that are graded at most as good as an alternative in [10] and the idea of depicting the possible positions of an alternative in [20], Liu et al., also defined the concept of extended preference map (EPM) from the perspective of the alternatives' position information.

Definition 2. We call $P_{j}=\left(p_{i, j}\right)_{n \rtimes \uparrow}$ the EPM of a linear ordinal ranking, where $I=\{1,2, \cdots ; n\}$ denotes the number of the alternatives, where $p_{i, j}$ denotes the extended preference map element (EPME) of the $A_{i}$ in $o_{j}$ provided by the $j_{t h}$ expert, if it satisfies: (1) $\forall i, p_{i, j} \in I$; (2) The cardinal of $p_{i, j}$ is 1, i.e., $\left|p_{i, j}\right|=1$; (3) The elements in $p_{i, j}$ represent the position of $A_{i}$ in $o_{j}$, and $p_{i, j}=1+n_{b, j}-n_{t, j}$, where $n_{b, j}$ denotes the number of alternatives that rank before $A_{i}$ in $o_{j}, n_{t, j}$ denotes the number of the symbol " $\sim$ " before $A_{i}$ in $o_{j}$.

Remark. If an expert has the same preference to all the alternatives, then, the EPME for each alternative is $\{1\}$.

The EVCS site selection process is full of risks [6,7] and experts have different reactions to gains and losses under risk environment. To better explain experts' reactions, the prospect theory proposed by Kaheman and Tversky [18] can be used. The value function is shown as follows $[18,39,41]$ :

$$
v(y)= \begin{cases}\left(y-y_{0}\right)^{\alpha}, & y>y_{0} \\ 0, & y=y_{0} \\ -\lambda\left(y-y_{0}\right)^{\beta}, & y<y_{0}\end{cases}
$$

where $y_{0}$ is the reference point, $\alpha$ and $\beta$ are positive real numbers and measure the curvature of the value function for gains and losses respectively, $\lambda$ is the coefficient of loss aversion. Generally, researchers assign the values to the 
different parameters as Tversky and Kahneman [9] advised, which are: $\alpha=\beta=0.88, \quad \lambda=2.25$.

\section{MAIN METHODS}

In this part, we present the main methods to solve the problems mentioned in Section I, We assume that there are $n$ alternative sites for EVCS in the finite set $\left\{A_{1}, A_{2}, \cdots, A_{i}, \cdots A_{n}\right\}$, and $m$ experts in the finite set $\left\{D_{1}, D_{2}, \cdots, D_{m}\right\}$.

\section{A. Inverse-prospect theory process for LORs}

When experts choose sites for EVCS in a risky environment, they become more sensitive to gains than losses and the process described by prospect theory automatically generates in their minds, and the evaluations are finally reflected by the LORs. That is to say, the LORs provided by experts are biased. Similarly, if we use EPMs to depict the LORs, the EPMs should also be taken as biased description. Hence, we should use the inverse value function of prospect theory to find the "true value". Given that there is no probability information in this situation, we just adjust the EPMs via inverse value function. Then, we can write the value function as Eq. (2).

$$
p_{i, j}=v\left(p_{i, j}^{*}\right)= \begin{cases}{\left[d\left(p_{i, j}^{*}, p_{0 j}\right)\right]^{\alpha},} & p_{i, j}^{*}>p_{0 j} \\ p_{i, j}^{*}, & p_{i, j}^{*}=p_{0 j} \\ -\lambda \times\left[d\left(p_{i, j}^{*}, p_{0 j}\right)\right]^{\beta}, & p_{i, j}^{*}<p_{0 j}\end{cases}
$$

where $v\left(p_{i, j}^{*}\right)$ denotes the results that have been processed by prospect theory, $p_{i, j}$ denotes the EPME of site $A_{i}$ evaluated by $D_{j}$ (in fact, $v\left(p_{i, j}^{*}\right)$ and $p_{i, j}$ are the same), $p_{i, j}^{*}$ denotes the true value of the EPME of site $A_{i}$ evaluated by $D_{j}, p_{0 j}$ denotes the reference point based on the evaluation of $D_{j}$, $d\left(p_{i, j}^{*}, p_{0 j}\right)$ denotes the distance between $p_{i, j}^{*}$ and $p_{0 j}$, where $d\left(p_{i, j}^{*}, p_{0 j}\right)=\left\{\begin{array}{l}p_{0 j}-p_{i, j}^{*}, p_{i, j}^{*}<p_{0 j} \\ p_{i, j}^{*}-p_{0 j}, p_{i, j}^{*}>p_{0 j}\end{array}\right.$.

Considering that the values of $p_{i, j}$ and $p_{i, j}^{*}$ should be located in $\left[1, \max p_{i, j}^{*}\right]$, we modify the function as follows:

$$
p_{i, j}=v\left(p_{i, j}^{*}\right)= \begin{cases}1, & p_{i, j}^{*}=1 \\ {\left[d\left(p_{i, j}^{*}, p_{0 j}\right)\right]^{\alpha},} & p_{i, j}^{*}>p_{0 j} \\ p_{i, j}^{*}, & p_{i, j}^{*}=p_{0, j} \\ -\lambda \times\left[d\left(p_{i, j}^{*}, p_{0, j}\right)\right]^{\beta}, & p_{i, j}^{*}<p_{0 j} \\ \max p_{i, j}^{*}, & p_{i, j}^{*}=\max p_{i, j}^{*}\end{cases}
$$

where $\max p_{i, j}^{*}$ denotes the maximum true value in the EPM provided by $D_{j}$. By Eq. (3), we get $\max p_{i, j}^{*}=\max p_{i, j}$.

To define the inverse value function, we determine the reference point $p_{0 j}$ and the distance between $p_{i, j}^{*}$ and $p_{0 j}$. Given that the evaluation information from the experts are all expressed by rankings and there are tie relationships existing in the rankings, we choose the medium position as the reference point and calculate it by:

$$
p_{0 j}=\frac{1+\max p_{i, j}^{*}}{2}=\frac{1+\max p_{i, j}}{2}
$$

Considering that all elements in EPMs are positive numbers, we change the parameter " $-\lambda$ " into " $\lambda$ ". It is obvious that $d\left(p_{i, j}^{*}, p_{0 j}\right) \in\left[0,\left(p_{0 j}-1\right)\right]$, when $p_{i, j}^{*}<p_{0 j}$, and $d\left(p_{i, j}^{*}, p_{0 j}\right) \in\left[0,\left(\max p_{i, j}^{*}-p_{0 j}\right)\right]$, when $p_{i, j}^{*}>p_{0 j}$. Then, the following formulas are satisfied (the values of $\alpha, \beta$ and $\lambda$ are determined as Tversky and Kahneman [9] advised):

$$
\begin{cases}v\left(p_{i, j}^{*}\right) \in\left[0,\left(p_{0 j}-1\right)^{0.88}\right], & p_{i, j}^{*}<p_{0 j} \\ v\left(p_{i, j}^{*}\right) \in\left[0, \lambda\left(p_{i, j}^{*}-p_{0 j}\right)^{0.88}\right], & p_{i, j}^{*}>p_{0 j}\end{cases}
$$

In addition, when we consider the range of $v\left(p_{i, j}^{*}\right)$, i.e., from the perspective of EPM, $p_{i, j}$ satisfies:

$$
\begin{cases}v\left(p_{i, j}^{*}\right) \in\left(1, p_{0 j}\right), & p_{i, j}^{*}<p_{0 j} \\ v\left(p_{i, j}^{*}\right) \in\left(p_{0 j}, \max p_{i, j}^{*}\right), & p_{i, j}^{*}>p_{0 j}\end{cases}
$$

Considering that, in this situation, the function should satisfy the conditions from these two perspectives at the same time, the range of $v\left(p_{i, j}^{*}\right)$ should be matched. To match the ranges of $v\left(p_{i, j}^{*}\right)$ from these two perspectives, we use linear map shown below:

$$
p_{i, j}=v\left(p_{i, j}^{*}\right)= \begin{cases}1, & p_{i, j}^{*}=1 \\ {\left[-\left(p_{0 j}-1\right)^{0.12}\right] \times\left(p_{0, j}-p_{i, j}^{*}\right)^{0.88}+p_{0 j},} & p_{i, j}^{*}<p_{0 j} \\ {\left[\frac{\left(\max p_{i, j}^{*}-p_{0 j}\right)^{0.2}}{\lambda}\right] \times\left[\lambda\left(p_{i, j}^{*}-p_{0 j}\right)^{0.88}\right]+p_{0 j},} & p_{i, j}^{*}>p_{0 j} \\ \max p_{i, j}^{*}, & p_{i, j}^{*}=\max p_{i, j}^{*}\end{cases}
$$

Based on Eq. (7), we can derive the inverse value function as

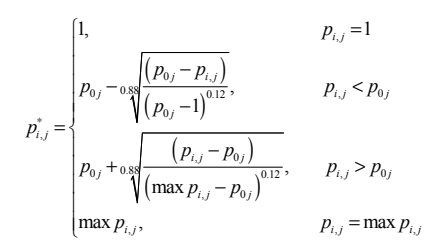

Then, we can use the results derived from Eq. (8) to calculate utility value of each site based on the EPMs provided by experts. The formula is modified as:

$$
u_{i, j}^{*}=\frac{\max p_{i, j}^{*}-p_{i, j}^{*}}{\max p_{i, j}^{*}-1}
$$

where $u_{i, j}^{*}$ denotes the utility value of site $A_{i}$ based on the EPM provided by $D_{j}$.

Then, the normalized utility value can be calculated by Eq. (10).

$$
u_{i, j}^{* N}=\frac{u_{i, j}^{*}}{\sum_{i=1}^{n} u_{i, j}^{*}}
$$

where $u_{i, j}^{* N}$ denotes the normalized utility value of the site $A_{i}$ based on the EPM provided by $D_{j}$. 


\section{B. The model that derives aggregated alternatives' weights}

After deriving the utility value of each site from each EPM, we focus on the method that derives the experts' weights, and the model that derive the aggregated sites' weights.

1) The method to determine the experts' weights: In order to aggregate the sites' weights from different experts, the weights of experts should be determined first. In this paper, we determine the weights of experts from the perspective of information energy. Since the concept of the information energy is measuring the non-fuzziness degree of information [23], we hold the view that the greater the information energy, the greater the internal differences in an expert's evaluation, and the easier to distinguish the differences between sites. In addition, considering that the LORs are expressed by different experts, we need to reduce the influence of the different average values among different experts to the most extent. Hence, we use coefficient of variation to calculate the information energy, which not only reflects the deviation degrees among the sites, but also reduces the influence of the different average values. The formula is shown as follows:

$$
I E_{j}=\frac{S\left(P_{j}\right)}{E\left(P_{j}\right)}
$$

where $I E_{j}$ denotes the information energy of the evaluation information of $D_{j}, S\left(P_{j}\right)=\sqrt{\frac{\sum_{i=1}^{n}\left(p_{i, j}^{*}-E\left(P_{j}\right)\right)^{2}}{n}}$ denotes the standard deviation of the EPM provided by $D_{j}, E\left(P_{j}\right)=\frac{\sum_{i=1}^{n} p_{i, j}^{*}}{n}$ denotes the average value of the EPM provided by $D_{j}$.

Then, since experts' preferences are more obvious with the greater information energy, we give the experts who have larger information energy larger weights. The weights of experts are calculated by Eq. (12).

$$
\sigma_{j}=\frac{I E_{j}}{\sum_{j=1}^{m} I E_{j}}
$$

where $\sigma_{j}$ denotes the weight of $D_{j}, I E_{j}$ denotes the information energy of the evaluation information of $D_{j}$.

2) The method to determine the aggregated alternatives' weights: To derive aggregated sites' weights, motivated by the method proposed by $\mathrm{Xu}$ [24], we construct the basic model as follows:

(Model 1)

$$
\begin{gathered}
\min z^{1}=\left(\sum_{i=1}^{n}\left|\omega_{i, j}-u_{i, j}^{* N}\right|^{2}\right)^{1 / 2} \\
\text { s.t. } \sum_{i=1}^{n} \omega_{i}=1 \\
\omega_{i} \in[0,1] \\
i=1,2, \cdots, n \\
j=1,2, \cdots, m
\end{gathered}
$$

where $\omega_{i, j}$ denotes the weight of site $A_{i}$ based on the EPM provided by $D_{j}, u_{i, j}^{* N}$ denotes the normalized utility value of the site $A_{i}$ based on the EPM provided by $D_{j}$. It is obvious that the model has solutions.

The objective of the model is actually consistency measurement. Considering that it is common to set a consistency index to judge whether the consistency degree can be accepted, we can modify Model 1 into Model 2 as follows:

$$
\begin{gathered}
\text { (Model 2) } \max z^{2}=\left(\sum_{i=1}^{n}\left|\omega_{i, j}-u_{i, j}^{* N}\right|^{2}\right)^{1 / 2} \\
\text { s.t. }\left(\sum_{i=1}^{n}\left|\omega_{i, j}-u_{i, j}^{* N}\right|^{2}\right)^{1 / 2}<C \\
\\
\sum_{i=1}^{n} \omega_{i}=1 \\
\omega_{i} \in[0,1] \\
i=1,2, \cdots, n \\
j=1,2, \cdots, m
\end{gathered}
$$

where $C$ is the consistency index that is up to experts.

It should be noted that the model may be unsolvable if the minimum value of $\left(\sum_{i=1}^{n}\left|\omega_{i, j}-u_{i, j}^{* N}\right|^{2}\right)^{1 / 2}$ is greater than $C$. In this situation, the LORs that cannot satisfy the consistency constraint would be deleted, meanwhile, in the experts' weights deriving process, the information energy of the corresponding experts would also be removed.

3) The model that aggregates the alternatives': In this paper, taking into consideration that there are many different experts giving their preferences, we attempt to establish the aggregation model to make the experts' preferences reach a consensus as much as possible.

$$
\begin{array}{r}
\min z^{3}=\sum_{i=1}^{n}\left(\sum_{j=1}^{m} \sigma_{j}\left(\omega_{i}-\omega_{i, j}\right)^{2}\right)^{1 / 2} \\
\text { s.t. } \sum_{i=1}^{n} \omega_{i}=1 \\
\omega_{i} \in[0,1] \\
i=1,2, \cdots, n \\
j=1,2, \cdots, m
\end{array}
$$

where $\omega_{i}$ denotes the weight of the site $A_{i}, \omega_{i, j}$ denotes the weight of the site $A_{i}$ based on the EPM provided by $D_{j}, \sigma_{j}$ denotes the weight of $D_{j}$.

Inspired by the concepts of the information energy, we attempt to add an objective in Model 3, which makes the model not only maximize the consensus degree, but also distinguish the deviation of different sites as much as possible. Therefore, Model 4 comes into being.

(Model 4)

$$
\begin{array}{r}
\min z^{41}=\sum_{i=1}^{n}\left(\sum_{j=1}^{m} \sigma_{j}\left(\omega_{i}-\omega_{i, j}\right)^{2}\right)^{1 / 2} \\
\max z^{42}=\left(\frac{1}{n} \sum_{i=1}^{n}\left(\omega_{i}-\frac{\sum_{i=1}^{n} \omega_{i}}{n}\right)^{2}\right)^{1 / 2} \\
\text { s.t. } \sum_{i=1}^{n} \omega_{i}=1 \\
\omega_{i} \in[0,1] \\
i=1,2, \cdots, n \\
j=1,2, \cdots, m
\end{array}
$$




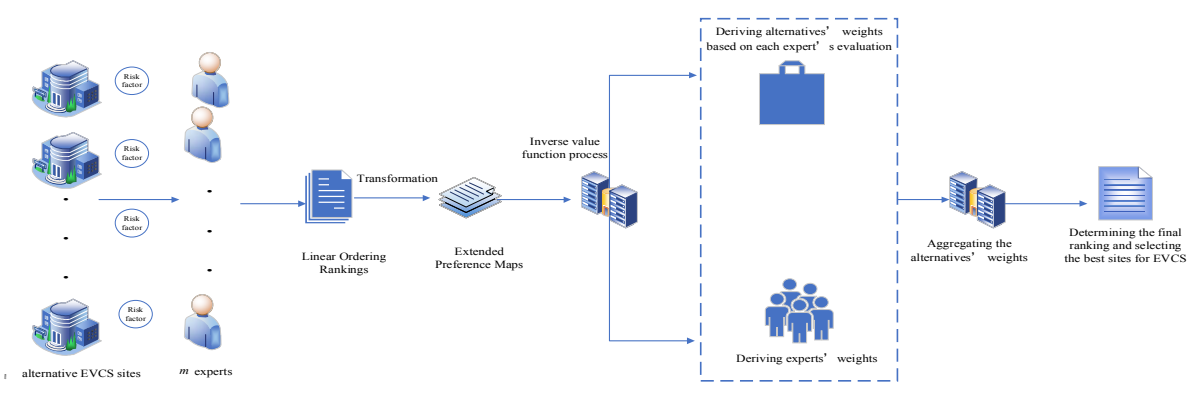

Fig. 1. The inverse prospect theory-based aggregation approach

The added objective is actually the standard deviation measure of $\omega_{i}$, in which the greater the standard deviation measure, the easier to distinguish the best EVCS site. Then, considering that weighted sum approach is one of the most classic way to transform double-objective models into singleobjective models, we use it to solve the double-objective model.

(Model 5)

$$
\begin{gathered}
\min z^{5}=\alpha \sum_{i=1}^{n}\left(\sum_{j=1}^{m} \sigma_{j}\left(\omega_{i}-\omega_{i, j}\right)^{2}\right)^{1 / 2}-(1-\alpha)\left(\frac{1}{n} \sum_{i=1}^{n}\left(\omega_{i}-\frac{\sum_{i=1}^{n} \omega_{i}}{n}\right)^{2}\right)^{1 / 2} \\
\text { s.t. } \sum_{i=1}^{n} \omega_{i}=1 \\
\quad \omega_{i} \in[0,1] \\
i=1,2, \cdots, n \\
j=1,2, \cdots, m
\end{gathered}
$$

From the model, we can derive the aggregated sites' weights, based on which we can adjust the order of the sites and select the best site for EVCS.

\section{The inverse prospect theory-based aggregation approach}

Based on the content mentioned above, the whole process of the method is presented as Fig. 1.

The whole process of the method mainly consists of four parts: 1) when experts express their preferences by LORs $n$ EVCS site selection process, the EPMs are utilized to depict the LORs; 2) Then, in order to decrease the influence of the risk reflection of experts, we use the inverse value function in the prospect theory to adjust the original EPMs; 3) based on the adjusted EPMs, the experts' weights and the sites' weights from the individual adjusted EPMs are derived. At this point, we utilize the information energy, which reflects the energy that distinguishes the alternatives from the individual evaluations; 4) Furthermore, we can derive the aggregated sites' weights through Model 5 and determine the final ranking. In this process, we consider the consensus and standard deviation at the same time.

The features of the approach are concluded as: (1) it considers the risk attitudes of experts, and use the inverse valuation function in the prospect theory to deal with the original information to find the "true value"; (2) determine experts' weights referring to the concept of the information energy, which provides a way for the determination of experts weights from a new perspective; (3) add the consistency constraint into the alternatives' weights deriving model, which relaxes the constraint of the model proposed by $\mathrm{Xu}$ [24]; (4) in the aggregation model, we consider the consensus degree and information energy at the same time, so that the results can maximize consensus degree and distinguish different alternatives as much as possible.

\section{ILLUSTRATIONS}

\section{A. Numerical case}

In this subsection, we assume that there are 7 alternative sites for EVCS $A=\left\{A_{1}, A_{2}, A_{3}, A_{4}, A_{5}, A_{6}, A_{7}\right\}, 15$ experts $D=\left\{D_{1}, D_{2}, D_{3}, D_{4}, D_{5}\right.$, $\left.D_{6}, D_{7}, D_{8}, D_{9}, D_{10}, D_{11}, D_{12}, D_{13}, D_{14}, D_{15}\right\}$ will select a site for EVCS. Firstly, experts provide their evaluations on the site selection

\begin{tabular}{|c|c|c|}
\hline DMs & LORs & Modified EPMs \\
\hline$D_{1}$ & $A_{2} \succ A_{3} \succ A_{6} \succ A_{7} \succ A_{1} \succ A_{4} \succ A_{5}$ & $\{4.861,1,2.108,5.892,7,3.139,4\}$ \\
\hline$D_{2}$ & $A_{2} \succ A_{4} \sim A_{3} \succ A_{7} \succ A_{1} \succ A_{5} \sim A_{6}$ & $\{3.910,1,2.090,2.090,5,5,3\}$ \\
\hline$D_{3}$ & $A_{4} \succ A_{3} \succ A_{2} \succ A_{1} \sim A_{6} \sim A_{5} \succ A_{7}$ & $\{3.910,3,2.090,1,3.828,3.828,5\}$ \\
\hline$D_{4}$ & $A_{4} \succ A_{2} \succ A_{3} \succ A_{5} \succ A_{7} \succ A_{6} \succ A_{1}$ & $\{7,2.108,3.139,1,4,5.892,5\}$ \\
\hline$D_{5}$ & $A_{3} \succ A_{5} \succ A_{7} \succ A_{1} \succ A_{4} \succ A_{2} \succ A_{6}$ & $\{4,5.892,1,4.861,2.108,7,3.139\}$ \\
\hline$D_{6}$ & $A_{7} \succ A_{5} \succ A_{6} \succ A_{3} \succ A_{2} \succ A_{1} \succ A_{4}$ & $\{5.892,4.861,4,7,2.108,3.139,1\}$ \\
\hline$D_{7}$ & $A_{5} \sim A_{3} \succ A_{2} \sim A_{1} \succ A_{6} \succ A_{7} \sim A_{4}$ & $\{2.070,2.070,1,4,1,2.930,4\}$ \\
\hline$D_{8}$ & $A_{6} \succ A_{7} \succ A_{3} \succ A_{1} \succ A_{4} \succ A_{2} \sim A_{5}$ & $\{3.902,6,3.099,4.899,6,1,2.101\}$ \\
\hline$D_{9}$ & $A_{1} \succ A_{7} \succ A_{5} \succ A_{3} \sim A_{4} \sim A_{6} \succ A_{2}$ & $\{1,5,3.910,3.910,3,3.910,2.090\}$ \\
\hline$D_{10}$ & $A_{3} \succ A_{1} \succ A_{4} \succ A_{7} \sim A_{6} \succ A_{5} \succ A_{2}$ & $\{2.101,6,1,3.099,4.899,3.901,3.901\}$ \\
\hline$D_{11}$ & $A_{6} \succ A_{2} \succ A_{1} \succ A_{5} \succ A_{7} \succ A_{3} \sim A_{4}$ & $\{3.099,2.101,6,6,3.902,1,4.900\}$ \\
\hline$D_{12}$ & $A_{5} \sim A_{1} \sim A_{4} \succ A_{2} \succ A_{7} \succ A_{6} \succ A_{3}$ & $\{1,2.090,5,1,1,3.910,3\}$ \\
\hline$D_{13}$ & $A_{7} \succ A_{4} \succ A_{2} \succ A_{1} \succ A_{3} \sim A_{5} \succ A_{6}$ & $\{3.902,3.099,4.899,2.101,4.899,6,1\}$ \\
\hline$D_{14}$ & $A_{1} \succ A_{7} \succ A_{2} \sim A_{3} \succ A_{4} \sim A_{5} \succ A_{6}$ & $\{1,3,3,3.910,3.910,5,2.090\}$ \\
\hline$D_{15}$ & $A_{4} \succ A_{2} \sim A_{3} \succ A_{5} \succ A_{6} \sim A_{7} \succ A_{1}$ & $\{5,2.090,2.090,1,3,3.910,3.910\}$ \\
\hline
\end{tabular}
for EVCS by LORs. After we transform the LORs provided by experts into EPMs, we use Eq. (9) to find the "true value" of the EPMs. The results are as Table 1.

TABLE I. THE LORS AND MODIFIED EPMS FROM DMS

Then, the experts' weights can be calculated: $\sigma_{1}=0.071$, $\sigma_{2}=0.066, \sigma_{3}=0.055, \sigma_{4}=0.071, \sigma_{5}=0.071, \sigma_{6}=0.071, \sigma_{7}=0.070$, $\sigma_{8}=0.067, \sigma_{9}=0.056, \sigma_{10}=0.064, \sigma_{11}=0.067, \sigma_{12}=0.089$, $\sigma_{13}=0.064, \sigma_{14}=0.057, \sigma_{15}=0.062$.

Further, we use Model 2 to calculate the weights of sites based on each expert's modified EPM, and let $C I=0.01$. Then, substituting the results into Model 5, we obtain the 
aggregated results as follows: $\omega_{1}=0.143, \omega_{2}=0.162, \omega_{3}=0.175$ , $\omega_{4}=0.142, \omega_{5}=0.124, \omega_{6}=0.098, \omega_{7}=0.155$. The final ranking is: $A_{3} \succ A_{2} \succ A_{7} \succ A_{1} \succ A_{4} \succ A_{5} \succ A_{6}$, which means that the site $A_{3}$ is the best site for the EVCS.

\section{B. Discussions}

In this subsection, we firstly make comparative analysis from the following three aspects: removing inverse value function from the approach, using Model 1 to derive sites' weights, and using the value function as the conventional way. Then, the sensitivity analysis is conducted, which mainly presents the influence of the parameter $\alpha$ in Model 5.

\section{1) Comparative analysis:}

a) Removing inverse value function from the approach: We remove the inverse value function from the approach and use the EPMs directly. In Fig. 2, the vertical axis denotes the sites' weights, and the horizontal axis elucidates that the sites' weights are calculated based on the information from $D_{j}, D_{j}^{\prime}(j=1,2, \cdots, 15)$ denotes the information which is not processed by inverse value function. From the figure, the differences between the sites' weights derived by the inverse value function and without inverse value function are not great. In this case, the inverse value function is used to modify the values and decrease the influence of the experts' risk reflection in the proposed method. Therefore, removing inverse value function has limited impact on the final rankings. In Fig. 3, the lines denote the results that are processed with and without inverse value function. The full line drops to 0 at $\sigma_{8}$ and $\sigma_{13}$, which denotes that the LORs provided by $D_{8}$ and $D_{13}$ are deleted since they cannot meet the consistency requirement. Besides, because we delete the LORs from the two experts, the weights of other experts increase, but the ranking among the experts' weights does not change, which is shown by the tendency of the line. From this perspective, we can see that the data processed by inverse value function can meet the consistency requirement better although the values of the rankings do not change a lot in this case. Further, the aggregated sites' weights from these two processes are different. The aggregated sites weights without inverse value function are: $\omega_{1}=0.149, \omega_{2}=0.176, \omega_{3}=0.189, \omega_{4}=0.116$, $\omega_{5}=0.142, \omega_{6}=0.095, \omega_{7}=0.133$, and the final ranking is $A_{3} \succ A_{2} \succ A_{1} \succ A_{5} \succ A_{7} \succ A_{4} \succ A_{6}$. Compared with the final ranking in the numerical case, the ranking positions of site $A_{3}$ and $A_{2}$ do not change, whereas the relationships between $A_{7}$ and $A_{1}$ , $A_{7}$ and $A_{5}, A_{4}$ and $A_{5}$ change obviously. As Fig. 3 and Fig. 4 show, the experts' weights and the sites' weights derived from individual EPMs do not change obviously. Through further observation, we find that in the LORs provided by $D_{8}$ and $D_{13}, A_{7}$ is better than $A_{1}$ and $A_{5}, A_{4}$ is better than $A_{5}$, particularly the $D_{13}$ ranks the site $A_{7}$ as the best one. If we delete the LORs provided by $D_{8}$ and $D_{13}$, the degrees that $A_{7}$ is superior than $A_{1}$ and $A_{5}$ descend, meanwhile, the degree that $A_{4}$ is superior than $A_{5}$ also descends. Therefore, in the final result of the numerical case, $A_{7}$ is superior than
$A_{1}$ and $A_{5}, A_{4}$ is superior than $A_{5}$, which is opposite in the result without inverse value function.

b) Using Model 1 to derive the sites' weights: In Fig. 4 , the vertical axis denotes the sites' weights, and the horizontal axis elucidates that the sites' weights are calculated based on the information form $D_{j}$, specifically $D_{j}^{\prime \prime}(j=1,2, \cdots, 15)$ denotes the information provided by $D_{j}$ is processed by Model 1 . From the figure, we can see that the result is similar to that in numerical case. That is to say, in this case, although in Model 2 we change the objective and add the consistency constraint into the model, the results can still maintain a good consistency that is close to the maximum consistency degree in Model 1.The final aggregated weights are: $\omega_{1}=0.147, \omega_{2}=0.162, \omega_{3}=0.172, \omega_{4}=0.139, \omega_{5}=0.123$, $\omega_{6}=0.1 \quad, \quad \omega_{7}=0.157 \quad$. The final ranking is $A_{3} \succ A_{2} \succ A_{7} \succ A_{1} \succ A_{4} \succ A_{5} \succ A_{6}$, which is identical to the ranking in numerical case. From this perspective, Model 2 can also obtain a sound result as Model 1 that pursues extremely consistent in this case. Besides, from the perspective of the model itself, it is possible that the value of the objective in Model 1 is greater than the consistency threshold, which will cause unreasonable results. Hence, the consistency measure is necessary in the site selection process.

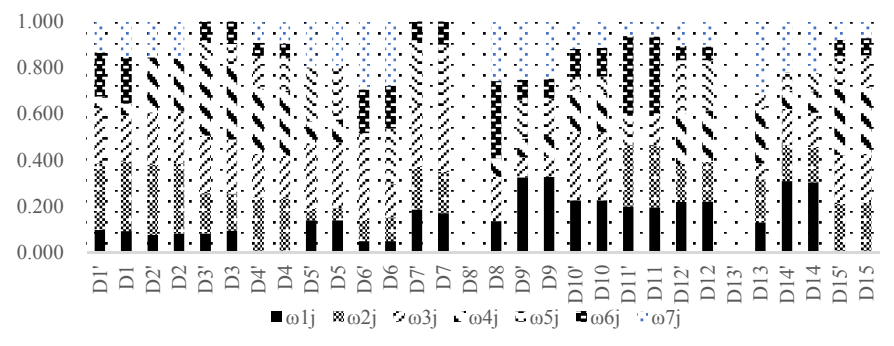

Fig. 2. The sites' weights derived from the model with inverse valuation and the model without inverse value function

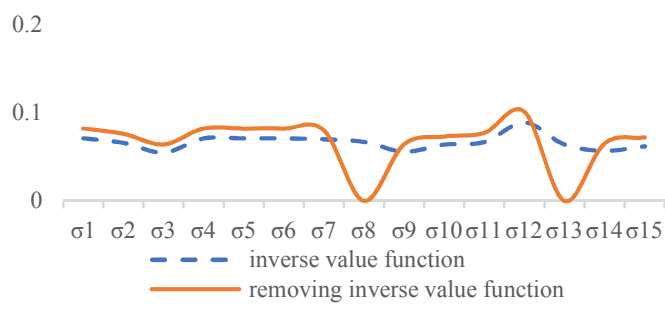

Fig. 3. The experts' weights derived from the model with inverse valuation and the model without inverse value function

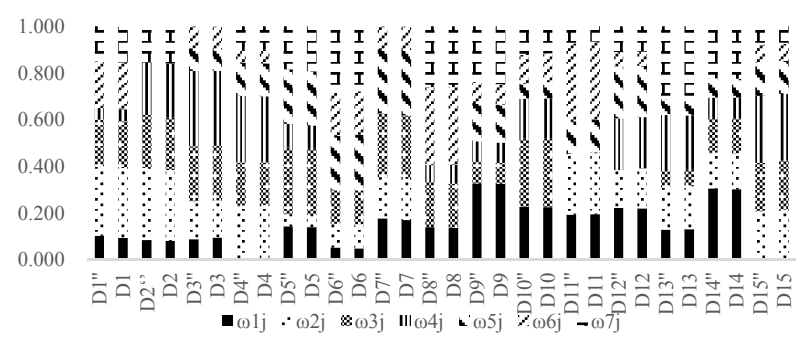

Fig. 4. The sites' weights derived from the model with consistency constraint and the model without consistency constraint 


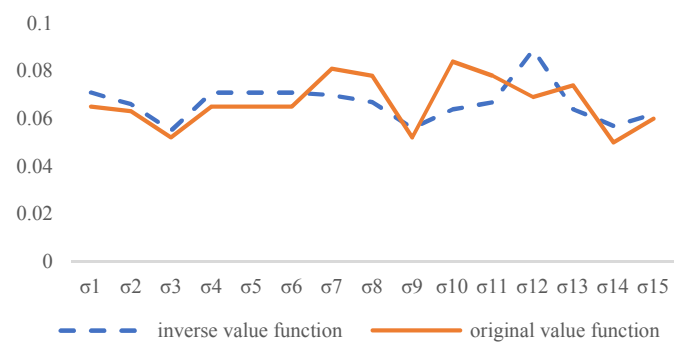

Fig. 5. The experts' weights derived from the method with the original value function and the method with inverse value function

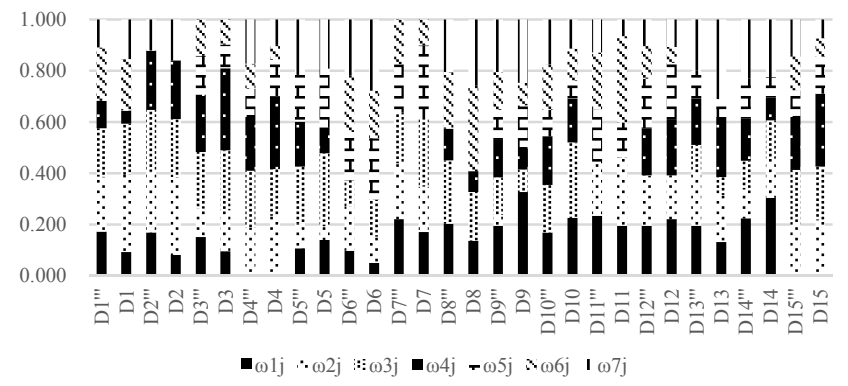

Fig. 6. The sites' weights derived from the model with the original value function and the model with inverse value function

c) Using prospect theory in the conventional way: In this part, we use the value function as the existing study [14], which just substitutes the values into the function directly. Fig. 5 shows the differences on the experts' weights in the situations that use the original value function and the inverse value function respectively. From the tendency of the lines, we can see that there are some changes occurring in the situation that uses the value function directly. The weights of the $D_{7}, D_{8}, D_{10}$ and $D_{13}$ have obvious increase, and the weight of the $D_{12}$ decreases obviously. In addition, as Fig. 6 shows, some changes also occur to the sites' weights. In order to know the difference of the sites' weights derived from these two ways, we use Euclidean distance formula to calculate the distance among sites. In addition, as Fig. 6 shows, some changes also occur to the sites' weights. In order to know the difference of the sites' weights derived from these two ways, we use Euclidean distance formula to calculate the distance among sites: $d_{j}^{*}=\left(\sum_{i=1}^{4}\left(\omega_{i, j}^{m}-\omega_{t, j}\right)^{2}\right)^{1 / 2}, j=1,2, \cdots, m$ where $d_{j}^{\prime \prime \prime}$ denotes the distance of the sites' weights derived from these two ways for $D_{j}, \omega_{i, j}^{\prime \prime}$ denotes the sites' weights that use the value function directly, $\omega_{i, j}$ denotes the sites' weights that use inverse value function. Fig. 7 shows the results of the distance of the sites' weights derived from these two ways. It is obvious that the greatest difference happens on the information from $D_{9}$, following is the $D_{11}$. From Fig. 5 and Fig. 6, we can see that for $D_{9}$, the difference is mainly reflected in $\omega_{1,9}^{\prime \prime \prime}$ and $\omega_{1,9}, \omega_{3,9}^{\prime \prime \prime}$ and $\omega_{3,9}, \omega_{4,9}^{\prime \prime \prime}$ and $\omega_{4,9}$, $\omega_{6,9}^{\prime \prime \prime}$ and $\omega_{6,9}$. For $D_{11}$, the difference is mainly reflected in $\omega_{1,11}^{\prime \prime \prime}$ and $\omega_{1,11}, \omega_{5,11}^{\prime \prime \prime}$ and $\omega_{5,11}, \omega_{6,11}^{\prime \prime \prime}$ and $\omega_{6,11}$, $\omega_{7,11}^{\prime \prime \prime}$ and $\omega_{7,11}$. The aggregated sites' weights using value function directly are: $\omega_{1}=0.164, \omega_{2}=0.149, \omega_{3}=0.165, \omega_{4}=0.139$, $\omega_{5}=0.107, \omega_{6}=0.127, \omega_{7}=0.148$, and the final ranking is $A_{3} \succ A_{1} \succ A_{2} \succ A_{7} \succ A_{4} \succ A_{6} \succ A_{5}$. Compared with the final ranking obtained by the method with inverse value function, the relationships between $A_{1}$ and $A_{2}, A_{1}$ and $A_{7}, A_{5}$ and $A_{6}$ change.

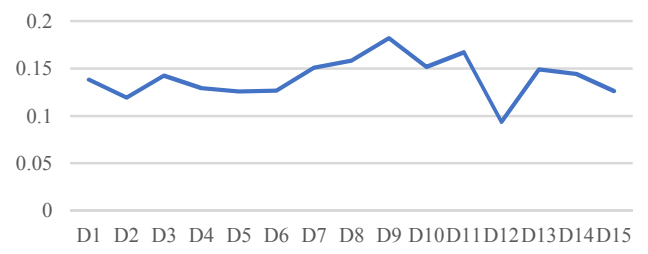

Fig. 7. The the distance of the sites' weights derived from the original value function and the method with inverse value function

\section{Sensitivity analysis}

Component heads identify the different components of your paper and are not topically subordinate to each other. Examples include Acknowledgments and References and, for these, the correct style to use is "Heading 5". Use "figure caption" for your Figure captions, and "table head" for your table title. Run-in heads, such as "Abstract", will require you to apply a style (in this case, italic) in addition to the style provided by the drop down menu to differentiate the head from the text.

In this part, we change the value of $\alpha$ from 0 to 1 with the step 0.05 in Model 5. The changes of aggregated sites' weights are shown as Fig .8. In the figure, we can see that when $\alpha$ changes from 0 to 0.15 , some extreme values occur, but the rankings start to become $A_{3} \succ A_{2} \succ A_{7} \succ A_{1} \succ A_{5} \succ A_{4} \succ A_{6}$, when $\alpha$ changes into 0.2 . Then, when $\alpha$ changes from 0.25 to 0.35, the aggregated ranking remains as $A_{3} \succ A_{2} \succ A_{7} \succ A_{4} \succ A_{1} \succ A_{5} \succ A_{6}$. Further, when $\alpha$ changes from 0.4 to 1 , the aggregated ranking remains as $A_{3} \succ A_{2} \succ A_{7} \succ A_{1} \succ A_{4} \succ A_{5} \succ A_{6}$. The top four alternatives have not changed, when $\alpha$ changes from 0.25 to 1 . Hence, from this perspective, the model is robust. In addition, given that $\alpha$ represents the importance degree of the consensus objective, the larger the value of $\alpha$, the more agreeable of the result. Hence, the value of $\alpha$ should better not be lower than 0.5 .

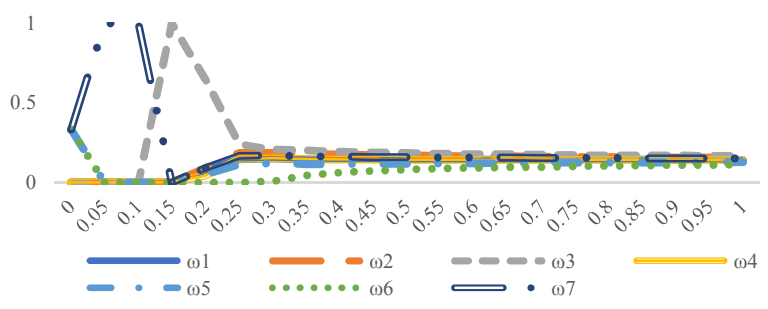

Fig. 8. Sensitivity analysis

From all above, we can see that 1) the inverse value function applied to this paper does not change the original LORs expressed by DMs. It mainly adjusts the values in EPMs, which are taken as the preference degrees in this paper. 2) The consistency constraint in Model 2 is a guarantee to keep the consistency degree of evaluation information. Besides, for the information that can reach extremely consistent, the model is equivalent to relaxing the constraint, which can reduce the unnecessary cost of reaching an 
absolute consistency. 3) The aggregated model is relatively robust when the value of the parameter $\alpha$ changes from 0.25 to 1 , and the model considers not only the consensus degree but also the information energy.

\section{CONCLUSION}

This paper proposes a new approach to aggregate LOR information and apply it to the site selection of EVCS. In the approach, the inverse value function in the prospect theory is used to reduce the influence of the risk in the EVCS site selection process. From the method analysis and the comparison analysis, we can see that the usage of the inverse value function can adjust the EPM to effectively reduce the influence of the risk. Meanwhile, through combining with the concept of information energy, the method to determine experts' weights is proposed. Then, during the process of deriving the alternatives' weights based on individual EPMs, we add the constraint of consistency to the model, which guarantees the consistency degree in an acceptable level, which guarantee the evaluation information in site selection process keep a good consistency. In addition, from the sensitivity analysis, we can see that the model is relatively robust. Hence, the aggregation approach proposed in this paper is applicable to the EVCS site selection. In the future, we can focus on the method that adjust the EPM that cannot meet the consistency constraint with low cost, which will be more meaningful to the site selection for EVCS. Additionally, considering that IE just determines the experts' weights from the perspective of information itself, cannot reflect the expertize level of the experts perfectly, we will attempt to combine the IE and other expertize level measures to determine the experts' weights.

\section{ACKNOWLEDGMENT}

This work was funded by the National Natural Science Foundation of China (Nos. 71571123, 71532007, 71771155).

\section{REFERENCES}

[1] Z. P. Liu, F. S. Wen, G. Ledwich, "Optimal Planning of Electric-Vehicle Charging Stations in Distribution Systems," IEEE Transactions on Power Delivery, vol. 28, pp. 102-110, 2013.

[2] S. Guo, H. Zhao, "Optimal site selection of electric vehicle charging station by using fuzzy TOPSIS based on sustainability perspective," Applied Energy, vol. 158, pp. 390-402, 2015.

[3] J. González, R. Alvaro, C. Gamallo, M. Fuentes, J. Fraile-Ardanuy, L. Knapen, D. Janssens, "Determining electric vehicle charging point locations considering drivers' daily activities," Procedia Computer Science, vol. 32, pp. 647-654, 2014.

[4] L. Jia, Z. C. Hu, Y. H. Song, Z. W. Luo, "Optimal siting and sizing of electric vehicle charging stations," Automation of Electric Power Systems, vol. 36, pp. 54-59, 2012.

[5] G. B. Wang, Z. Xu, F. S. Wen, K. P. Wong, "Traffic-constrained multiobjective planning of electric-vehicle charging stations," IEEE Transactions on Power Delivery, vol. 28, pp. 2363-2372, 2013.

[6] J. C. Liu, Q. S. Wei, "Risk evaluation of electric vehicle charging infrastructure public-private partnership projects in China using fuzzy TOPSIS," Journal of Cleaner Production, vol. 189, pp. 211-222, 2018.

[7] J. Dong, G. Y. Xue, B. J. Wang, "Risk evaluation of charging facilities of electric vehicles based on fuzzy analytic network process," Advanced Materials Research, vols. 1070-1072, pp. 1600-1608, 2015.

[8] X. J. Gou, Z. S. Xu, H. C. Liao, "Group decision making with compatibility measures of hesitant fuzzy linguistic preference relations," Soft Computing, vol. 23, pp. 1511-1527, 2019.

[9] C. Trepel, C. R. Fox, R. A. Poldrack, "Prospect theory on the brain? Toward a cognitive neuroscience of decision under risk," Cognitive brain research, vol. 23, pp. 34-50, 2005.

[10] T. González-Arteaga, J. C. R. Alcantud, R. de Andrés Calle, “A new consensus ranking approach for correlated ordinal information based on
Mahalanobis distance," Information Sciences, vol. 372, pp. 546-564, 2016.

[11] F. Franceschini, D. Maisano, L. Mastrogiacomo, "A new proposal for fusing individual preference orderings by rank-ordered agents: A generalization of the Yager's algorithm," European Journal of Operational Research, vol. 249, pp. 209-223, 2015.

[12] F. Franceschini, D. A. Maisano, and L. Mastrogiacomo, "A novel algorithm for fusing preference orderings by rank-ordered agents," Elsevier North-Holland, Inc. 2015

[13] R. R. Yager, "Fusion of multi-agent preference orderings," Fuzzy Sets \& Systems, vol. 117, pp. 1-12, 2001.

[14] H. M. Liang, W. Xiong, Y. C. Dong, “A prospect theory-based method for fusing the individual preference-approval structures in group decision making." Computers \& Industrial Engineering, vol. 117, pp. 237-248, 2018.

[15] F. Franceschini, D. Maisano, L. Mastrogiacomo, "Fusion of multi-agent preference orderings in an ordinal semi-democratic decision-making framework," Measurement, vol. 91. pp. 699-702, 2016.

[16] X. Z. Xu, J. M. Martel, B. F. Lamond, "A multiple criteria ranking procedure based on distance between partial preorders," European Journal of Operational Research, vol. 133, pp. 69-80, 2001.

[17] J. Borda, "Mémoire sur les élections au scrutin," Histoire de l'Academie Royale des Sciences pour 1781, 1784.

[18] F. Chiclana, F., Herrera, E. Herrera-Viedma, "Integrating three representation models in fuzzy multipurpose decision making based on fuzzy preference relations," Fuzzy sets and Systems, vol. 97, pp. 33-48, 1998.

[19] Y. M. Wang, J.-B. Yang, D.-L. Xu, "A preference aggregation method through the estimation of utility intervals," Computers \& Operations Research, vol. 32, pp. 2027-2049, 2005.

[20] F. J. Hou, E. Triantaphyllou, "An iterative approach for achieving consensus when ranking a finite set of alternatives by a group of experts," European Journal of Operational Research, vol. 275, pp. 570$579,2019$.

[21] M. S. Beg, N. Ahmad, "Soft computing techniques for rank aggregation on the world wide web," World Wide Web, vol. 6, pp. 5-22, 2003

[22] P. Diaconis, "Group Representations in Probability and Statistics," Lecture notes-monograph series, vol. 11, pp. i-192, 1988.

[23] D. Dumitrescu, "A definition of an informational energy in fuzzy sets theory," Information and Control, vol. 22, pp. 57-59, 1977.

[24] Z. S. Xu, X. Q. Cai, S. S. Liu, "Nonlinear programming model integrating different preference structures," IEEE Transactions on Systems, Man, and Cybernetics-Part A: Systems and Humans, vol. 41, pp. 169-177, 2010.

[25] E. J. Emond, D. W. Mason, “A new rank correlation coefficient with application to the consensus ranking problem," Journal of Multi criteria Decision Analysis, vol. 11, pp. 17-28, 2002.

[26] W. D. Cook, M. Kress, L. M. Seiford, “An axiomatic approach to distance on partial orderings," RAIRO - Operations Research, vol. 20, pp. $115-122,1986$ 